\title{
A Message Propagation Model for Hybrid Vehicular Communication Protocols*
}

\author{
Anna Maria Vegni ${ }^{1}$, and Thomas D.C. Little ${ }^{2}$ \\ ${ }^{1}$ Department of Applied Electronics \\ University of Roma Tre, Rome, Italy \\ 2 Department of Electrical and Computer Engineering \\ Boston University, Boston, Massachusetts \\ amvegni@uniroma3.it,tdcl@bu.edu
}

May 27, 2010

MCL Technical Report No. 05-27-2010

\begin{abstract}
This paper addresses a hybrid approach on message propagation in vehicular networks. Short-range Vehicle-to-Vehicle (V2V) communications cannot always guarantee connectivity to the network cloud, especially when traffic density is very low. By utilizing existing wireless network infrastructure it is possible to greatly enhance the capabilities of short range V2V technologies. Such existing and future communications infrastructure includes UMTS, HSDPA, Wi-Fi, and WiMax.

For this multi-network environment we analyze how messages are forwarded by vehicles communicating via a hybrid protocol exploiting both short-range V2V communication schemes and Vehicle-to-Infrastructure (V2I) concepts. The protocol switching from V2V to V2I, and vice versa, occurs through a decision algorithm that considers observed network state. We characterize the bounds of information propagation and compare performance with traditional message propagation based on opportunistic networking. Simulation results show the effectiveness of the proposed hybrid vehicular communication protocol.
\end{abstract}

*In Proc. 2nd International Workshop on Communication Technologies for Vehicles (Nets4Cars 2010), Newcastle, UK, July 2010. This work is supported, in part, by the Engineering Research Centers Program of the National Science Foundation under NSF Cooperative Agreement No. EEC-0812056. Any opinions, findings and conclusions or recommendations expressed in this material are those of the author(s) and do not necessarily reflect those of the National Science Foundation. 


\section{Introduction}

Vehicular Ad-hoc Networks (VANETs) are emerging as the preferred network design for Intelligent Transportation Systems (ITS) based on, for example, Dedicated Short-Range Communications (DSRC) [1]. Vehicle-to-Vehicle (V2V) communications are supported due to smart vehicles equipped by on-board computers with multiple network interface cards (e.g., Wi-Fi, HSDPA, GPS), and emerging wireless technologies (e.g., IEEE 802.11p, WiMax, LTE).

Although $\mathrm{V} 2 \mathrm{~V}$ is potentially the most viable approach to low-latency short-range vehicular networks, connectivity between vehicles may not always be available due to quick topology network changes, vehicle speed, and traffic density [1]. In sparsely connected and totally disconnected scenarios, vehicles are not always able to communicate each other, and V2V does not represent the most appropriate interconnection scheme for some applications [2], especially non-safety-critical ones.

A solution to longer-range vehicular connectivity should consider preexisting network infrastructure such as wireless access points (called as Road-Side Units, RSUs). Communications between vehicles and RSUs are supported by Vehicle-to-Infrastructure (V2I) protocol. V2I limitations are due to particular vehicular applications required. Moreover, performance is strictly dependent on the specific wireless technology for the RSUs.

In order to achieve the advantages of both V2V and V2I protocols, we propose a hybrid vehicular protocol, called as Vehicle-to-X (V2X). Based on V2V and V2I, V2X supports traditional VANET scenario with a heterogeneous network environment, and aims for vehicles $(i)$ to communicate between them (via V2V), and (ii) to connect to the Internet (via V2I). As a result, V2X permits hybrid vehicular communications that means each vehicle can switch from V2V to V2I, and vice versa, on the basis of a protocol switching decision algorithm.

In this paper we illustrate the behavior of V2X protocol, and analyze how information is propagated. In our approach, we assume a data push communication model, in which information messages are propagated via localized (limited range) broadcast.

The paper is structured as follows: Section 2 deals with related work in message propagation in VANETs, while in Section 3 we describe the main criterion on which V2X is based. In Section 4 we give definitions about different data propagation rates obtained with V2X, while Section 5 describes the main phases of V2X protocol. Simulation results are shown in Section 6. We compare the performance of the $\mathrm{V} 2 \mathrm{X}$ protocol with respect to traditional opportunistic networking technique (i.e., V2V) in terms of message dissemination. Finally, conclusions are drawn in Section 7.

\section{Related Work}

Due to different traffic scenarios (i.e., dense, sparse or totally disconnected traffic neighborhoods [3]), vehicles in VANETs move in clusters and form interconnected blocks of vehicles [4]. As a consequence, vehicular connectivity is not always available, and messages can be lost or never received.

Opportunistic forwarding is applied in VANETs in order to achieve connectivity between vehicles, and to disseminate information, [4-6]. Message propagation occurs through links built dynamically, where any vehicle can be used as next hop and subsequent hops forward the message to the final destination. $\mathrm{V} 2 \mathrm{~V}$ communications exploit connectivity from other neighboring vehicles by a bridging technique [6]. 


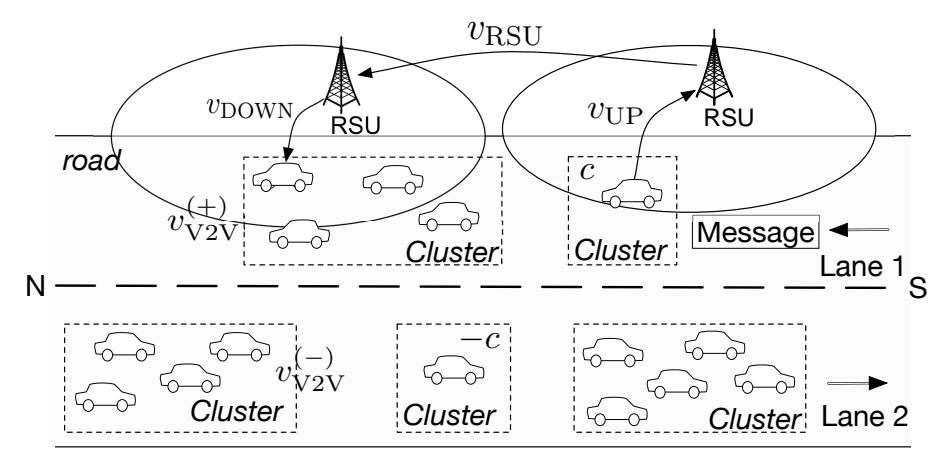

Figure 1: VANET scenario with heterogeneous wireless network infrastructure.

Many authors have addressed the analysis of message propagation in VANETs. In [7] Resta et al. deal with multi-hop emergency message dissemination through a probabilistic approach. The authors derive lower bounds on the probability that a vehicle correctly receives a message within a fixed time interval. Similarly, Jiang et al. [8] introduce an efficient alarm message broadcast routing protocol, and estimate the receipt probability of alarm messages sent to vehicles. Other works [9-11] analyze the message propagation model on the basis of the main VANET characteristics such as number of hops, vehicle position, mobility, etc. Yousefi et al. [9] consider a single-hop dissemination protocol based on Quality-of-Service metrics, while Chen et al. [10] propose a robust message dissemination technique based on the vehicles position. Finally, Nadeem et al. [11] present a data dissemination model based on bidirectional mobility of paths between a couple for vehicles. In all previous works data traffic is disseminated only through vehicles communicating via V2V. No network infrastructure nor V2I protocol have been considered.

The use of a vehicular grid together with an infrastructure has been discussed in [12] and [13], where benefits of using the opportunistic infrastructure placed on the roads are analyzed. Our approach relies on the network scenario depicted by Gerla et al. in [12], but we propose a novel protocol that provides switching from V2V to V2I, and vice versa. For such a technique we expect that the message propagation via $\mathrm{V} 2 \mathrm{X}$ be improved by a correct use of vehicular communication protocols (i.e., V2V and V2I).

\section{Vehicle-to-X Technique}

V2X enables vehicles to communicate via V2V or V2I on the basis of a protocol switching decision. We now describe the main aspects of this decision criterion.

Let us consider a cluster $C$ comprised of a set $S$ of vehicles (i.e., $S=\{1,2, \ldots, n\}$ ). Then, $m$ Road-Side Units (RSUs) (i.e., $m<n$ ) are displaced in the network scenario as depicted in Figure 1. Each vehicle is able to communicate via V2V on the basis of a fixed transmission range radio model [14]. We assume that only a limited subset of vehicles in the cluster $C$, (i.e., $S^{\prime}=\{1,2, \ldots, l\} \subset S$, with $l<n$ ), is able to connect to an RSU via V2I. For example, not all the vehicles might have an appropriate network interface card, and/or are not in the range of connectivity of an RSU. Analogously, we assume that only $k$ RSUs (i.e., $k=\{1,2, \ldots, h\}$ with $h<m$ ) are available to V2I communications.

For the connectivity link from the $i$-th to the $j$-th vehicle we define as link utilization time 
$q_{(i, j)}[\mathrm{s}]$ the time needed to transmit a message of length $L$ [bit] from the $i$-th to the $j$-th vehicle, at an actual data rate $f_{(i, j)}[\mathrm{Mbit} / \mathrm{s}]$, such as

$$
q_{(i, j)}=\frac{L}{f_{(i, j)}} .
$$

For a link between a $i$-th vehicle and an $k$-th RSU, the data rate is obtained by the nominal data rate $\breve{f}_{(i, k)}$ by applying a Data Rate Reduction (DRR) factor $\left(\right.$ i.e., $\left.\rho_{(i, k)}\right)$ that depends on the distance from the vehicle to the RSU, namely $f_{(i, k)}=\rho_{(i, k)} \breve{f}_{(i, k)}$. The $D R R$ factor increases when a vehicle is laying within the bound of a wireless cell.

Let us define a path from the $i$-th vehicle to the $k$-th RSU comprised of a sequence of $M$ hops, where a single hop represents a link between two neighboring vehicles. The path length represents the number of hops $M$ for a single path. It follows that the maximum number of directed links from a vehicle to an RSU is $\alpha=l \cdot h$, while the maximum number of different paths that can connect the $i$-th vehicle to the $k$-th $\mathrm{RSU}$ is $n \cdot \alpha$.

From the definition of path, we define the path utilization time $Q_{(i, k)}$ [s] from the $i$-th vehicle to the $k$-th RSU as the sum of single link utilization time parameters $\left(\right.$ i.e., $\left.q_{(i, j)}\right)$, for each hop that comprises the path, as

$$
Q_{(i, k)}=q_{(i, j)}+q_{(j, x)}+\ldots+q_{(x, k)}=L \sum_{\substack{i=1 \\ x \in S}}^{n}\left[f_{(i, x)}^{-1}\right]
$$

The optimal path will be the one, among all the paths $n \alpha$, with the minimized path utilization time, such as

$$
\min _{s=1,2, \ldots, n \alpha} Q_{(i, k)}^{(s)}=L \cdot \min _{s=1,2, \ldots, n \alpha} \sum_{\substack{i=1 \\ x \in S}}^{n}\left[f_{(i, x)}^{(s)}\right]^{-1} .
$$

Equation (3) is compared with the link utilization times in V2V communications in order to detect the most appropriate vehicular protocol.

\section{Data Propagation Rates}

In this section we illustrate how a message is propagated in a VANET incorporating a heterogeneous network infrastructure. For our purposes, we give several definitions of message dissemination rates for different cases.

Figure 1 depicts a vehicular network scenario in which a wireless network infrastructure partially covers the VANET. Vehicles move in clusters in two separated lanes (i.e., lane 1, and 2), where north (i.e., $\mathrm{N}$ ), and south (i.e., S) represent the directions of lane 1, and 2, respectively. The message propagation direction is assumed for this case to be $\mathrm{N}$.

Local connectivity information is received by each vehicle through broadcast "hello" messages, and establish if a vehicle is within a cluster or is traveling alone on the road. In contrast, a vehicle will know if there are neighboring wireless networks on the basis of broadcast signaling messages sent by the RSUs.

We assume the vehicles are traveling at a constant speed $c[\mathrm{~m} / \mathrm{s}]$, while $v[\mathrm{~m} / \mathrm{s}]$ is the message propagation rate within a cluster, such as $v=x / t$, where $x$ is the transmission range distance 
between two consecutive and connected vehicles (i.e., $0<x \leq 125 \mathrm{~m},[14]$ ), and $t$ [s] is the time necessary for a successful transmission, which depends on the single link of connected vehicles. As a consequence, the average message propagation rate within a cluster (i.e., $v[\mathrm{~m} / \mathrm{s}]$ ) should consider each single contribution due to each single link $(i, j)$, such as

$$
v=\frac{1}{h} \sum_{i, j} v_{(i, j)}=\frac{1}{h} \sum_{i, j} \frac{x_{(i, j)}}{q_{(i, j)}}=\frac{1}{h L} \sum_{i, j} x_{(i, j)} \cdot f_{(i, j)},
$$

where $v_{(i, j)}[\mathrm{m} / \mathrm{s}]$ is the message propagation rate for the link $(i, j)$, and $h$ is the number of hops occurred within a cluster. The message propagation rate $v[\mathrm{~m} / \mathrm{s}]$ depends on the average message propagation rate for each hop within a cluster and increases for a low number of hops $h$.

Now, let us consider $v_{\mathrm{RSU}}[\mathrm{m} / \mathrm{s}]$ as the message propagation rate within the network infrastructure, as

$$
v_{\mathrm{RSU}}=d \cdot \frac{B}{L},
$$

where $d$ is the distance between two consecutive RSUs. The ratio between the message length $L$ [bit], and the effective data rate $B$ [bit/s], for the link between the $m$-th and $(m+1)$-th RSU, represents the time necessary to forward a message between two consecutive RSUs.

It follows that $v_{\mathrm{RSU}}$ is strictly dependent on the message propagation direction. A message is forwarded to an RSU if it is placed along the same message propagation direction (see Figure 1). An RSU that receives a message by a vehicle can therefore forward it to the next RSU. The potential for communications between RSUs is introduced in this work in order to avoid connectivity interruptions caused by low traffic densities, and that the V2V protocol cannot always solve, [5].

Equation (5) represents the message propagation rate within the preexisting network infrastructure. We also consider the message propagation rate in uplink (downlink), when a vehicle sends a message to an RSU (and vice versa), such as:

$$
v_{\mathrm{UP}}=\frac{x_{r}}{L} \cdot \tilde{f}_{(i, m)}, \quad v_{\mathrm{DOWN}}=\frac{x_{r}}{L} \cdot \tilde{f}_{(m, i)},
$$

where $x_{r}$ is the distance that separates the $i$-th vehicle and the $m$-th RSU, while $\tilde{f}$ is the effective transmission data rate for the link $(i, m)$ (uplink), and $(m, i)$ (downlink), respectively. From (5) and (6), it follows that the message propagation rate $v_{\mathrm{V} 2 \mathrm{I}}[\mathrm{m} / \mathrm{s}]$ for communication between vehicles and RSUs via V2I depends on the effective transmission data rates in uplink and downlink, and on the effective data rate for intra RSU communications, such as

$$
\begin{aligned}
v_{\mathrm{V} 2 \mathrm{I}} & =v_{\mathrm{UP}}+v_{\mathrm{RSU}}+v_{\mathrm{DOWN}}= \\
& =\frac{1}{L}\left[d \cdot B+x_{r} \cdot\left(\tilde{f}_{(i, m)}+\tilde{f}_{(m, i)}\right)\right] .
\end{aligned}
$$

As an analogy, we define the message propagation rate for communications via $\mathrm{V} 2 \mathrm{~V}$ (i.e., $\left.v_{\mathrm{V} 2 \mathrm{~V}}[\mathrm{~m} / \mathrm{s}]\right)$, as

$$
v_{\mathrm{V} 2 \mathrm{~V}}^{( \pm)}= \pm(v+c)
$$

which depends on the constant velocity $c$ of vehicles and on the effective transmission data rates within a cluster $C$ according to (4). The positive or negative sign of $v_{\mathrm{V} 2 \mathrm{~V}}$ depends on the message propagation direction.

Finally, when no connectivity occurs (i.e., a vehicle is traveling alone), the message propagation rate is equal to $\pm c$, which depends on message propagation direction.

We characterize the behavior of the whole system in terms of six transition states as follows: 
1. Messages are traveling along on a vehicle in the $\mathrm{N}$ direction at speed $c[\mathrm{~m} / \mathrm{s}]$;

2. Messages are propagating multi-hop within a cluster in the $\mathrm{N}$ direction at speed $v_{\mathrm{V} 2 \mathrm{~V}}^{(+)}[\mathrm{m} / \mathrm{s}]$;

3. Messages are traveling along a vehicle in the $\mathrm{S}$ direction at speed $-c[\mathrm{~m} / \mathrm{s}]$;

4. Messages are propagating multi-hop within a cluster in the $\mathrm{S}$ direction at speed $v_{\mathrm{V} 2 \mathrm{~V}}^{(-)}[\mathrm{m} / \mathrm{s}]$;

5. Messages are transmitted via radio by an RSU in the $\mathrm{N}$ direction at speed $v_{\mathrm{V} 2 \mathrm{I}}[\mathrm{m} / \mathrm{s}]$;

6. Messages are transmitted via radio by an RSU in the $\mathrm{S}$ direction at speed $-v_{\mathrm{V} 2 \mathrm{I}}[\mathrm{m} / \mathrm{s}]$.

States $(1-4)$ are typical for data propagation with an opportunistic networking techniques in VANET scenarios in which vehicles communicate only via V2V [6], while states 5, and 6, have been added for vehicles communicating via V2I. All the six states can occur when vehicles communicate via V2X.

As illustrated in [6], the bridging technique is strongly employed in opportunistic networking for vehicular networks in order to avoid disconnections. In VANETs, there are two message propagation direction (i.e., forward and reverse propagation). In forward message propagation, each vehicle is assumed to travel in the $\mathrm{N}$ direction at speed $c[\mathrm{~m} / \mathrm{s}]$, and the message is propagated in the $\mathrm{N}$ direction. The message propagation rate has a minimum value due to the speed of the vehicle (i.e., $c[\mathrm{~m} / \mathrm{s}]$ ) since the message is traveling along the vehicle. When a connection between two consecutive vehicles traveling in the $\mathrm{N}$ direction is available, the message will be propagated via $\mathrm{V} 2 \mathrm{~V}$ at a rate $v_{\mathrm{V} 2 \mathrm{~V}}^{(+)}$.

Moreover, if no vehicle connection is available, the bridging technique can attempt to forward a message to clusters along the S (opposite) direction whenever they are overlapping with the cluster along the $\mathrm{N}$ direction [6]. From such considerations, we evince the following definitions for forward and reverse message propagation rates, respectively.

Definition (Forward Message Propagation rate): the forward message propagation rate, when a vehicle is communicating via $V 2 \mathrm{~V}$, is in the range $\left[c, v_{\mathrm{V} 2 \mathrm{~V}}^{(+)}\right]$. In contrast, when a vehicle communicates via V2I, the forward message propagation rate is in the range $\left[c, v_{\mathrm{V} 2 \mathrm{I}}\right]$.

Definition (Reverse Message Propagation rate): the reverse message propagation rate, when a vehicle communicates via $\mathrm{V} 2 \mathrm{~V}$, is in the range $\left[-c, v_{\mathrm{V} 2 \mathrm{~V}}^{(-)}\right]$, while for vehicles communicating via $V 2 I$, the range of reverse message propagation rate is $\left[-c, v_{\mathrm{V} 2 \mathrm{I}}\right]$.

A message can be forwarded by vehicles traveling in an opposite direction respect to the message propagation. In this case each vehicle is assumed to travel along the $\mathrm{S}$ direction at speed $-c[\mathrm{~m} / \mathrm{s}]$ along with the message. When a connection between two consecutive vehicles traveling in the $\mathrm{S}$ direction is available, the message will be propagated via $\mathrm{V} 2 \mathrm{~V}$ at a rate $v_{\mathrm{V} 2 \mathrm{~V}}^{(-)}$. Instead, when no vehicle connection is available, a message will be forwarded to some clusters along the $\mathrm{N}$ (opposite) direction, similarly to bridging technique in forward message propagation. 


\section{V2X Algorithm}

This Section illustrates the algorithm for protocol switching decisions in V2X. It is mainly based on (i) the Infrastructure Connectivity (IC) parameter, which gives information if a vehicle is able to connect to an RSU, and on (ii) the optimal path detection technique. The pseudo-code is depicted in Algorithm 1. The algorithm accepts one input (i.e., the vehicle's IC), and returns the actual message propagation rate (i.e., $\left.\left\{v_{\mathrm{V} 2 \mathrm{~V}}, v_{\mathrm{V} 2 \mathrm{I}}\right\}\right)$.

Let us assume a source vehicle $A$ is communicating with other vehicles (relay) via $\mathrm{V} 2 \mathrm{~V}$ in a sparsely connected neighborhood where the transmission range distance between two consecutive vehicles is under the connectivity bound (i.e., $x \leq 125 \mathrm{~m}$, [14]). The vehicle $A$ is not inside a wireless network (i.e., IC =0). A destination vehicle $B$ is driving far away from $A$, and other vehicles (relay) are available to communicate each other. In such scenario, the proposed algorithm should work according to two main tasks, such as (i) checking IC parameter, and (ii) tracking the destination vehicle(s).

Every time a vehicle forwards a message it checks its IC value. When IC $=1$, the vehicle calculates the optimal path according to (3) in order to send the message directly to the selected RSU via V2I. Otherwise the vehicle forwards the message to neighboring vehicles via V2V. Then, the RSU knows the destination vehicle's position (i.e., by A-GPS). If the destination vehicle is traveling within the RSU's wireless coverage, the RSU will send the message directly to the destination vehicle. Otherwise, the RSU will be simply forwarding the message to the RSU that is actually managing the vehicle's connectivity. Finally, the message will be received by the destination vehicle.

\section{Simulation Results}

We present simulation results that verify the effectiveness of our approach as compared with traditional opportunistic networking scheme in VANET.

As a measure of performance, we calculate the average message displacement $[\mathrm{m}]$ in VANETs via V2X. In each of the six states illustrated in Section 4, the message displacement is a function

of time, varying for different traffic scenarios, speed and network conditions (e.g., in state 1 the message displacement is $c \cdot t$ ).

We simulated a typical vehicular network scenario by the following events: $(i)$ at $t=0 \mathrm{~s}$ a source vehicle is traveling in the $\mathrm{N}$ direction and sends a message along on the same direction (state 1); (ii) at $t=2 \mathrm{~s}$ the message is propagated multi-hop within a cluster in the $\mathrm{N}$ direction (state 2); (iii) at $t=6 \mathrm{~s}$ a relay vehicle enters an RSU's radio coverage, and the message is transmitted via V2I to the RSU, until it will be received by other vehicles at $t=10 \mathrm{~s}$ (state 5 ).

We compared this scenario with traditional opportunistic networking technique in VANETs [6], where the following events occur: $(i)$ at $t=0 \mathrm{~s}$ a source vehicle traveling in the $\mathrm{N}$ direction sends a message along on the same direction (state 1); (ii) at $t=4 \mathrm{~s}$ the message is forwarded to a vehicle in the $\mathrm{S}$ direction (state 3); (iii) at $t=6 \mathrm{~s}$ the message propagates via multi-hop within a cluster in the $\mathrm{N}$ direction (state 2). The transmission stops at $t=10 \mathrm{~s}$. For comparative purposes, in the simulation setup we posed parameters according to [6] and [15], including $c=20 \mathrm{~m} / \mathrm{s}$ and $d=500 \mathrm{~m}$. Typical message size $L=300 \mathrm{bit}$, data rate transmission $B=10 \mathrm{Mbit} / \mathrm{s}$ (e.g., for WiMax connectivity), and $x_{r}=400 \mathrm{~m}$ have been assumed. The transmission rates in DSRC have 




Algorithm 1: Protocol switching decisions in V2X.

been assumed equal to $6 \mathrm{Mbit} / \mathrm{s}$ [1]. For each hop in a cluster (i.e., $h=5$ ) we considered different distances between couples of vehicles (i.e., 100, 75, 50, 40, and $30 \mathrm{~m}$ ).

Figure 2 (left) depicts the maximum/minimum message propagation bounds for V2X protocol in forward message propagation mode. We notice a strong increase in the message propagation with respect to other forms of opportunistic networking: after $t=10 \mathrm{~s}$, the message has been propagating for approximately $30 \mathrm{~km}$ in V2X (Figure 2 (left)), while only $1.5 \mathrm{~km}$ in traditional V2V (Figure 2 (right)). This is due to the protocol switching decision of V2X which exploits high data rates from wireless network infrastructure, while V2V is limited to DSRC protocol.

Analogously, a message can be forwarded in reverse message propagation by vehicles traveling in an opposite direction (Figure 3). In this case, the message propagation rates are in the range $\left[-c,-v_{\mathrm{V} 2 \mathrm{I}}\right]$ and $\left[-c, v_{\mathrm{V} 2 \mathrm{~V}}^{(-)}\right][\mathrm{m} / \mathrm{s}]$, for $\mathrm{V} 2 \mathrm{X}$ and traditional opportunistic networking scheme, respectively. Again, while V2X assures high values for message displacement (i.e., at $t=10 \mathrm{~s}$, a message has been propagated up to around $70 \mathrm{~km}$, as shown in Figure 3 (left)), traditional V2V can achieve low values (i.e., at $t=10 \mathrm{~s}$, messages have reached $1.3 \mathrm{~km}$ far away from the source vehicle, as depicted in Figure 3 (right)). Small fluctuations of message displacement in forward and reverse cases with $\mathrm{V} 2 \mathrm{X}$ (i.e. 50 , and $70 \mathrm{~km}$ ) depend on traffic density, and RSUs' positions. 

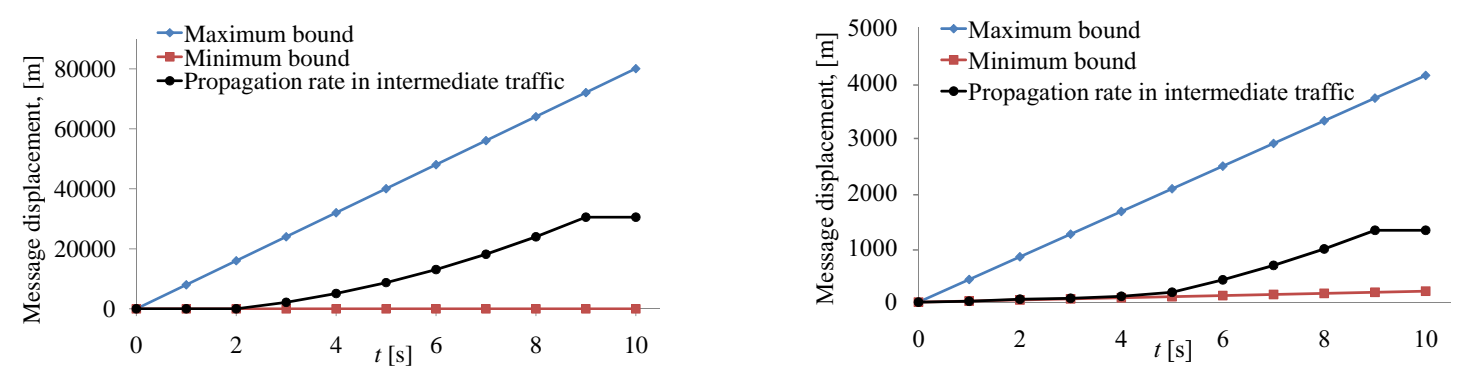

Figure 2: Forward message propagation with (left) V2X protocol, (right) traditional opportunistic networking.
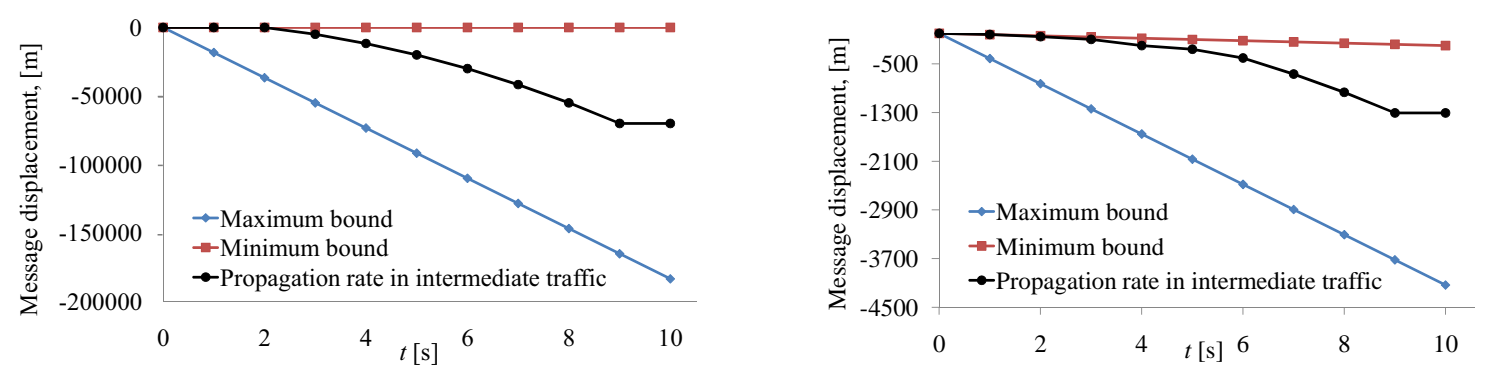

Figure 3: Reverse message propagation with (left) V2X protocol, (right) traditional opportunistic networking.

\section{Conclusions}

In this work we described a hybrid vehicular communication protocol V2X and the mechanism by which a message can be propagated under this technique. V2X exploits both traditional V2V and V2I techniques, through the use of fixed infrastructure (i.e. Road Side Units).

In this heterogeneous VANET scenario, we have characterized the upper and lower bounds for message propagation rates. Simulation results — in terms of message displacement - have shown how V2X protocol improves network performance, with respect to traditional opportunistic networking technique applied in VANETs.

Future work will address to V2X complexity analysis (i.e. protocol switching decision delay), and statistical distribution on message arrival rates.

\section{References}

[1] G. Held, Inter-and intra-vehicle communications. C CRC Press, November 8, 2007.

[2] B. D. Chiara, F. Deflorio, and S. Diwan, "Assessing the effects of inter-vehicle communication systems on road safety," Intelligent Transport Systems, IET, vol. 3, no. 2, pp. 225-235, June 2009. 
[3] O. Tonguz, N. Wisitpongphan, F. Bai, P. Mudalige, and V. Sadekar, "Broadcasting in VANET," in Proc. on Mobile Networking for Vehicular Environments, Anchorage, AK, May 2007, pp. 7-12.

[4] L. Pelusi, A. Passarella, and M. Conti, "Opportunistic networking: data forwarding in disconnected mobile ad hoc networks," IEEE Communications Magazine, vol. 44, no. 11, pp. 134-141, November 2006.

[5] X. Ma, X. Chen, and H. Refai, "Performance and Reliability of DSRC Vehicular Safety Communication: A Formal Analysis," EURASIP Journal on Wireless Comm. and Networking, Special Issue on Wireless Access in Vehicular Environments, no. 3, p. 13, January 2009.

[6] T.D.C. Little, and A. Agarwal, "An Information Propagation scheme for VANETs," in Proc. on 8th International IEEE Conference on Intelligent Transportation Systems, September 2005, pp. 155-160.

[7] G. Resta, P. Santi, and J. Simon, "Analysis of multihop emergency message propagation in vehicular ad hoc networks," in Proc. on the 8th ACM International Symposium on Mobile Ad Hoc Networking and Computing, Montreal, September 09-14, 2007, pp. 140-149.

[8] H. Jiang, H. Guo, and L. Chen, "Reliable and Efficient Alarm Message Routing in VANET," in Proc. on the 28th International Conference on Distributed Computing Systems Workshops, 2008, pp. 186-191.

[9] S. Yousefi, M. Fathy, and A. Benslimane, "Performance of beacon safety message dissemination in Vehicular Ad hoc NETworks (VANETs)," Journal of Zhejiang University Science A, 2007.

[10] W. Chen, R. Guha, T. Kwon, J. Lee, and Y. Hsu, "A survey and challenges in routing and data dissemination in vehicular ad hoc networks," in IEEE International Conference on Vehicular Electronics and Safety, Columbus, USA, September 22-24, 2008.

[11] T. Nadeem, P. Shankar, and L. Iftode, "A comparative study of data dissemination models for VANETs," in Proc. on the 3rd Annual International Conference on Mobile and Ubiquitous Systems, San Jose, (California), July 17-21, 2006, pp. 1-10.

[12] M. Gerla, B. Zhou, Y.-Z. Leey, F. Soldo, U. Leey, and G. Marfia, "Vehicular Grid Communications: The Role of the Internet Infrastructure," in Proc. on Wireless Internet Conference, Boston, August 2006.

[13] G. Marfia, G. Pau, E. D. Sena, E. Giordano, and M. Gerla, "Evaluating Vehicle Network Strategies for Downtown Portland: Opportunistic Infrastructure and Importance of Realistic Mobility Models," in Proc. of MobiOpp 2007, Porto Rico, June, 2007.

[14] A. Agarwal, and T.D.C. Little, “Access Point Placement in Vehicular Networking," in Proc. of 1st International Conference on Wireless Access in Vehicular Environments, Dearborn, USA, December 8-9, 2008. 
[15] H. Wu, R. Fujimoto, and G. Riley, "Analytical Models for Information Propagation in Vehicle-to-Vehicle Networks," in Proc. on ACM VANET, Philadelphia, USA, October, 2004. 